\title{
Use of Xpert MRSA/SA BC for direct testing on breast milk from women with mastitis: a diagnostic accuracy study
}

\author{
Niamh Cahill', Haydn Hammerton' ${ }^{1}$, Ciara O'Connor ${ }^{1}$, Marian Brennan ${ }^{2}$, Maeve Eogan ${ }^{3}$ and Richard J. Drew $^{4,5,6 *}$ \\ ${ }^{1}$ Department of Microbiology, Rotunda Hospital, Dublin 1, Ireland \\ ${ }^{2}$ Department of Midwifery, Rotunda Hospital, Dublin 1, Ireland \\ ${ }^{3}$ Department of Obstetrics, Rotunda Hospital, Dublin 1, Ireland \\ ${ }^{4}$ Clinical Innovation Unit, Rotunda Hospital, Dublin 1, Ireland \\ 5rish Meningitis and Sepsis Reference Laboratory, Temple Street Children's University Hospital, Dublin 7, Ireland \\ ${ }^{6}$ Royal College of Surgeons in Ireland, Dublin 2, Ireland
}

\begin{abstract}
The purpose of this pilot diagnostic accuracy study was to determine if the Xpert MRSA/SA BC (Cepheid, USA) test kit which is used on the GeneXpert (Cepheid, USA) platform for blood cultures, could be used to directly test breast milk samples. The advantage of a rapid PCR test over culture is that results would be available in 2 hours, rather than 48 hours by traditional culture methods and its benefit has been shown for positive blood cultures. A hundred consecutive breast milk samples were included in the study taken during 2015-2017. The reference standard test was breast milk culture using traditional agar plate methods.

Overall, 30\% ( $n=30)$ of the samples had S.aureus cultured and all were detected by the PCR test (Sensitivity 100\%, 95\% Confidence Interval 88.4-100\%). Three (10\%) of the positive culture samples had MRSA detected on culture and all were MRSA positive by GeneXpert as well. Of the 70 culture negative samples, 12 (17\%) were positive for MSSA by PCR and 58 (83\%) were negative (Specificity 82.8\%, 95\% CI 71.97-90.82\%). Importantly the negative predictive value was 100\%.

This study has shown that the Xpert MRSA/SA BC panel can be used for direct testing of milk samples from post-partum women with severe mastitis. The three cases of MRSA infection were correctly identified with no false positive results. Use of this platform would allow affected women to receive effective antimicrobial treatment at the point of admission rather than potentially delaying correct treatment for 48 hours while awaiting culture results. A larger interventional trial is warranted to determine the potential clinical impact of this test on antimicrobial stewardship as some cases of mastitis may not have been infectious or the pathogen may not have been S.aureus.
\end{abstract}

\section{Introduction}

Mastitis is a common postpartum infection in lactating women. When women present to hospital, often they have been treated in the community using oral $\beta$-lactam antimicrobials without success [1]. This, therefore, raises concerns about the presence of methicillin resistant Staphylococcus aureus (MRSA) and consideration of treating the patient with antimicrobials to specifically target MRSA [2]. The purpose of this pilot diagnostic accuracy study was to determine if the Xpert MRSA/SA BC (Cepheid, USA) test kit which is used on the GeneXpert (Cepheid, USA) platform for blood cultures, could be used to directly test breast milk samples. The advantage of this PCR test over culture is that results would be available in 2 hours, rather than 48 hours by traditional culture methods and its benefit has been shown for positive blood cultures $[3,4,5]$. This would allow for earlier prescribing of effective antimicrobials in cases of MRSA infection, and to confidently withhold MRSA treatment in those who test MRSA negative by PCR.

\section{Materials and methods}

This was a prospective diagnostic accuracy study comparing the detection of S.aureus in breast milk by culture method and molecular method using the Xpert MRSA/SA BC test cartridge. Inclusion criteria were any breast milk sample sent to the laboratory for culture from an in-patient who was admitted with severe mastitis. The requesting of breast milk samples for culture was standard practice in the hospital as part of the mastitis care pathway. A hundred consecutive breast milk samples were included in the study taken during 2015-2017. The study took place in the Rotunda Hospital, which is a stand-alone maternity hospital with approximately 8,500 live births per year.

The reference standard test was breast milk culture. A swab was dipped in to the EBM sample and inoculated on to a Columbia blood agar plate which was incubated in $5-10 \%$ CO2 for $40-48$ hours at $35+/-2^{\circ} \mathrm{C}$. A second swab was dipped in to the EBM sample and inoculated on to a Colorex MRSA chromogenic agar plate (EO Labs, United Kingdom). This plate was incubated in air for 18-24 hours at $35+/-2^{\circ} \mathrm{C}$. All Agar plates were reviewed, and suspect colonies were

Correspondence to: R Drew, Department of Microbiology, Rotunda Hospital, Dublin 1, Ireland, Tel: 353-1-8171700, E-mail: rdrew@rotunda.ie

Key words: MRSA, mastitis, pregnancy, lactation, GeneXpert

Received: April 13, 2018; Accepted: April 20, 2018; Published: April 26, 2018 
identified using the Vitek Maldi system (BioMerieux, France). Growth of S.aureus was reported as either 'detected' or 'not detected' by culture. Susceptibility testing was performed in line with EUCAST methods on the Vitek-2 system (BioMerieux, France).

For the study, $3-5 \mathrm{ml}$ of the sample was transferred into a sterile universal container, labelled with a study number. $50 \mu \mathrm{L}$ of EBM was transferred into an elution reagent vial. The vial was then closed and vortexed for 10 seconds. The MRSA/SA Blood Culture cartridge lid was opened. Using a sterile pipette all contents of the elution vial were aspirated and transferred to the S -Chamber of the MRSA/SA Blood culture cartridge. The chamber was closed and immediately loaded on to the GeneXpert and PCR run started. Xpert MRSA/SA Blood culture cartridge package insert instructions, precautions and warnings were followed. The results were recorded as 'detected' or 'not detected' for both S.aureus and MRSA. The laboratory scientists performing the culture and the PCR test were not blinded to the results, and they were aware that the patients had been admitted with severe mastitis. There was no time delay in sample processing as both the culture and PCR testing were done on the same day.

The sensitivity and specificity of the PCR method was compared to the culture method using the MedCalc statistical software [Medcalc, version 16.4.3]. There were no intermediate results as both results for the PCR and culture were binary results. There was no missing data in the study. The sample size of 100 was based on a convenient sample size given the infrequency of admissions with severe mastitis. No patient information was retained as this was part of the ethics permission that was granted. No data was available regarding prior exposure to antimicrobials.

\section{Results}

The results for the study are shown in figure 1 , and $30 \%(n=30)$ of the samples had S.aureus cultured. Three samples had MRSA detected on culture and all were MRSA positive by GeneXpert as well. There were no false positive MRSA samples reported on the GeneXpert. The sensitivity of the test was $100 \%$ with a specificity of $82.86 \%$ (table 1 ). There were no adverse events reported as this was a non-interventional study.

\section{Conclusion}

This study has shown that the Xpert MRSA/SA BC panel can be used for direct testing of milk samples from post-partum women with severe mastitis. The three cases of MRSA infection were correctly identified with no false positive results. Use of this platform would allow affected women to receive effective antimicrobial treatment at the point of admission rather than potentially delaying correct treatment for 48 hours while awaiting culture results. Eighty-eight percent of patients had a PCR result which matched the culture result for S.aureus, and importantly this would have been available within 2 hours. The PCR positive, culture negative cases may be due to pre-testing antimicrobial exposure and future studies evaluating the use of this test should incorporate recording of antimicrobial exposure pre-treatment as this was a limitation of this project. There is no likely bias in this project as the samples were consecutive and had no inclusion criteria other than severe mastitis requiring hospitalisation.

The advantage of introducing this PCR test for routine testing of breast milk samples is that it can quickly identify or outrule the

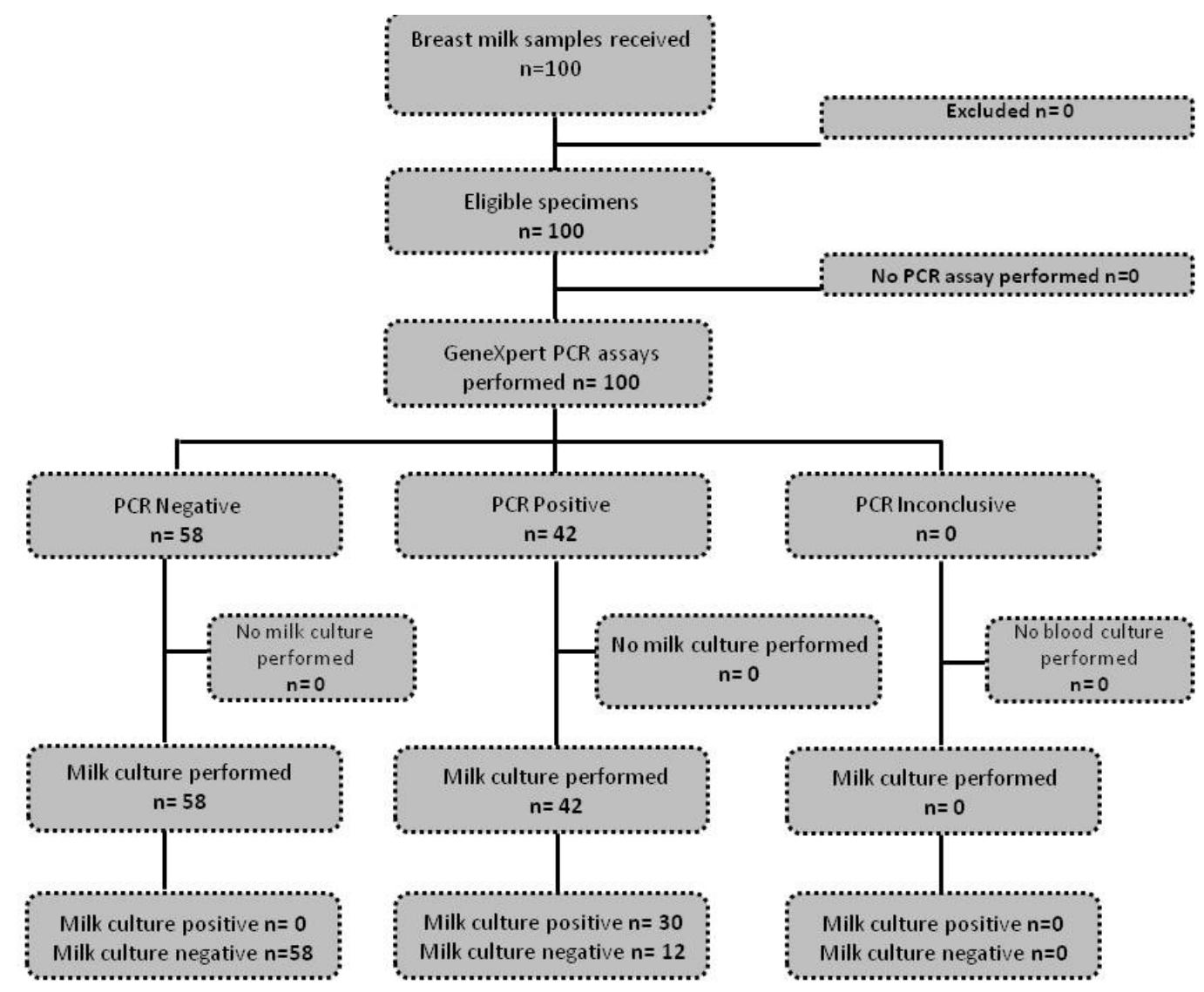

Figure 1. 
Table 1. Comparison of culture for S.aureus on breast milk samples to molecular testing with GeneXpert platform using Xpert MRSA/SA BC test kit.

\begin{tabular}{|c|c|c|c|c|c|}
\hline \multirow{2}{*}{} & & \multicolumn{4}{c|}{ Genexpert } \\
\hline \multirow{3}{*}{ Culture } & MRSA & MSSA & Negative & Total \\
\cline { 2 - 6 } & MSSA & 3 & 0 & 0 & \\
\cline { 2 - 6 } & MSSA & 0 & 27 & 0 & 100 \\
\hline \multirow{2}{*}{ Total } & Negative & 0 & 12 & 58 & \\
\hline \multicolumn{5}{|c|}{ Comparison of GeneXpert to culture for detection of S.aureus } \\
\hline \multicolumn{5}{|c|}{ Value } & $95 \%$ Confidence Interval \\
\hline \multicolumn{3}{|c|}{ Sensitivity } & $100 \%$ & $88.43-100$ \\
\hline \multicolumn{3}{|c|}{ Specificity } & $82.86 \%$ & $71.97-90.82$ \\
\hline \multicolumn{3}{|c|}{ Area under curve } & 0.91 & $0.84-0.96$ \\
\hline Positive predictive value & $71.43 \%$ & $55.42-84.28$ \\
\hline Negative predictive value & $100 \%$ & $93.84-100 \%$ \\
\hline
\end{tabular}

MSSA= methicillin susceptible Staphylococcus aureus, MRSA= methicillin resistant Staphylococcus aureus

presence of MRSA in patients with severe mastitis. By having this test quickly available to clinicians, it would be possible to ensure that both MRSA infected patients receive vancomycin or linezolid at the point of admission, and also that non-MRSA infected women do not receive unnecessary vancomycin treatment while awaiting cultures.

\section{Acknowledgements}

We would like to thank the staff of the Microbiology Laboratory at the Rotunda hospital for their assistance during the study

\section{Funding}

This study was supported by a financial grant from the Friends of the Rotunda Foundation

Conflict of Interest: None of the authors have a conflict of interest to declare

\section{References}

1. Jahanfar S, Ng CJ, Teng CL (2016) Antibiotics for mastitis in breastfeeding women. Sao Paulo Med J 134: 273. [Crossref]

2. Farahnik B, Murase JE (2016) Antibiotic safety considerations in methicillin-resistant Staphylococcus aureus postpartum mastitis. J Am Acad Dermatol 75:e149. [Crossref]

3. Davies J, Gordon CL, Tong SY, Baird RW, Davis JS (2012) Impact of results of a rapid Staphylococcus aureus diagnostic test on prescribing of antibiotics for patients with clustered gram-positive cocci in blood cultures. J Clin Microbiol. 50:2056-2058. [Crossref]

4. Spencer DH, Sellenriek P, Burnham CA (2011) Validation and implementation of the GeneXpert MRSA/SA blood culture assay in a pediatric setting. Am J Clin Pathol. 136:690-4. [Crossref]

5. Zboromyrska Y, De la Calle C, Soto M, Sampietro-Colom L, Soriano A, et al. (2016) Rapid Diagnosis of Staphylococcal Catheter-Related Bacteraemia in Direct Blood Samples by Real-Time PCR. Plos One 11: e0161684. [Crossref]

Copyright: @2018 Cahill N. This is an open-access article distributed under the terms of the Creative Commons Attribution License, which permits unrestricted use, distribution, and reproduction in any medium, provided the original author and source are credited. 